\title{
ARTICLE
}

\section{Medial canthoplasty for the management of exposure keratopathy}

\author{
Robi N. Maamari ${ }^{1} \cdot$ Philip L. Custer $^{1} \cdot$ Michael G. Neimkin $^{1,2} \cdot$ Steven M. Couch ${ }^{1}$
}

Received: 14 October 2018 / Accepted: 22 December 2018 / Published online: 1 February 2019

(c) The Royal College of Ophthalmologists 2019

\begin{abstract}
Purpose To report the surgical technique and functional outcomes of the medial canthoplasty for the treatment of exposure keratopathy.

Patients/Methods An IRB approved, retrospective review of patients who underwent medial canthoplasty for exposure keratopathy was performed. Patient demographics, reported symptoms, and clinical examination findings were collected and analyzed from pre-operative and follow-up visits.

Results The study included 73 consecutive cases in which the medial canthoplasty was performed in patients with exposure keratopathy. The average follow-up period was 7.9 months (median: 4.7 months; range: 1-150 months). Complete or partial improvement in ocular symptoms (dryness; pain/irritation; tearing) was achieved in 95\% (69/73). Clinically, 85\% (41/48) of patients demonstrated a post-operative reduction in lagophthalmos and 90\% (60/67) showed improvement in ocular surface findings. Complications were rare (1/73) and reversal of medial canthoplasty was not required in any case.

Conclusions The medial canthoplasty appears to be a safe and effective technique to narrow the palpebral fissure, provide lower eyelid support, and improve keratopathy. It is an uncomplicated procedure that may be considered for the treatment of exposure keratopathy caused by facial paralysis and lower eyelid malposition.
\end{abstract}

\section{Introduction}

Exposure keratopathy is a well-known ocular complication in conditions with eyelid paralysis and malposition $[1,2]$. Common causes leading to the development of exposure keratopathy include facial nerve palsy, eyelid laxity, previous periocular surgery, globe prominence, and anterior lamellar shortening causing cicatricial ectropion [3-5]. Lagophthalmos and poor eyelid mechanics associated with these conditions can result in significant morbidities ranging from ocular irritation and discomfort to corneal infection, ulceration, and scarring.

Supplementary information The online version of this article (https:// doi.org/10.1038/s41433-019-0347-9) contains supplementary material, which is available to authorized users.

Steven M. Couch

CouchS@wustl.edu

1 Department of Ophthalmology and Visual Sciences, Washington University School of Medicine, St. Louis, MO, USA

2 Present address: Ophthalmic Plastic and Cosmetic Surgery, LLC, Atlanta, GA, Georgia
Multiple surgical techniques have been described to treat the various causes of ocular exposure and lower eyelid abnormalities [6-17]. Ideally, surgical planning should not only correct the anatomic deformity but also be complimented with ease of procedure, acceptable cosmesis, and few complications. While different variations of the medial canthoplasty procedures have previously been reported, the literature describing functional post-operative outcomes remains limited [18-20]. In this article, we present a medial canthoplasty technique modified from that reported by Dr. Otis Lee for management of exposure keratopathy and report outcomes when employed as a standalone surgical intervention [18].

\section{Methods}

A retrospective review was performed including patients who underwent medial canthoplasty between April 2000 and February 2018. All procedures were performed by attending oculoplastic surgeons (SMC and PLC). Indications for surgery included ocular symptoms and exposure keratopathy caused by facial palsy, lower eyelid retraction, keratoconjunctivitis sicca, or neurotrophic keratopathy. Patient demographics, associated procedures, and 
complications were reviewed. Additionally, clinical data (visual acuity, lagophthalmos measurement, slit-lamp corneal keratopathy examination [Grade: 0 to 4]), and patientreported symptoms in three categories (tearing, dryness, and pain/irritation) from pre-operative and follow-up visits were collected and analyzed. If bilateral procedures were performed, each eye was assessed as an independent case. Patients who were treated with additional simultaneous procedures (lateral tarsal strip, medial spindle, permanent tarsorrhaphy, upper eyelid weight, etc.) to address the exposure keratopathy were excluded from this study. Informed consent was obtained from all participants included in the study. This study was approved by the Institutional Review Board of Washington University in St. Louis and adhered to the tenets of the Declaration of Helsinki.

\section{Surgical technique}

The medial canthoplasty may be performed in the office procedure room under local anesthesia or in the operating room with monitored anesthetic sedation (Video 1). A limited amount of subcutaneous lidocaine with epinephrine is administered at the medial canthal region in order to minimize anatomical distortion. An incision is made at the upper eyelid mucocutaneous junction starting $1 \mathrm{~mm}$ medial to the upper punctum and extended to the medial canthal angle. A second mucocutaneous incision is created medial to the lower punctum and extended to meet the prior incision (Fig. 1a). Skin flaps are then elevated overlying the medial canthal tendons. A thin strip of mucosa of the upper and lower eyelids is excised to expose the upper and lower medial canthal tendons with care taken to avoid canalicular damage (Fig. 1b). The upper and lower medial canthal tendons are then adjoined using two or three interrupted 6-0 or 7-0 polyglactin sutures. These sutures are passed through the superior palpebral portion of the orbicularis muscle, then through the upper and lower medial canthal tendons with shallow passes to avoid damage to the canalicular system, and finally through the inferior palpebral orbicularis muscle (Fig. 1c). The skin flaps are closed with interrupted 6-0 gut sutures (Fig. 1d).

\section{Results}

The study included 73 procedures performed on 46 patients with an average age of 69 years (range: 19-93 years). A bilateral medial canthoplasty was performed for 27 patients (54 cases) and 19 patients were treated with a unilateral canthoplasty. Of the 73 cases, $39(53.4 \%)$ cases were performed on female patients. The average follow-up period was 7.9 months (median: 4.7 months; range: 1150 months). The indications for surgery included of lower

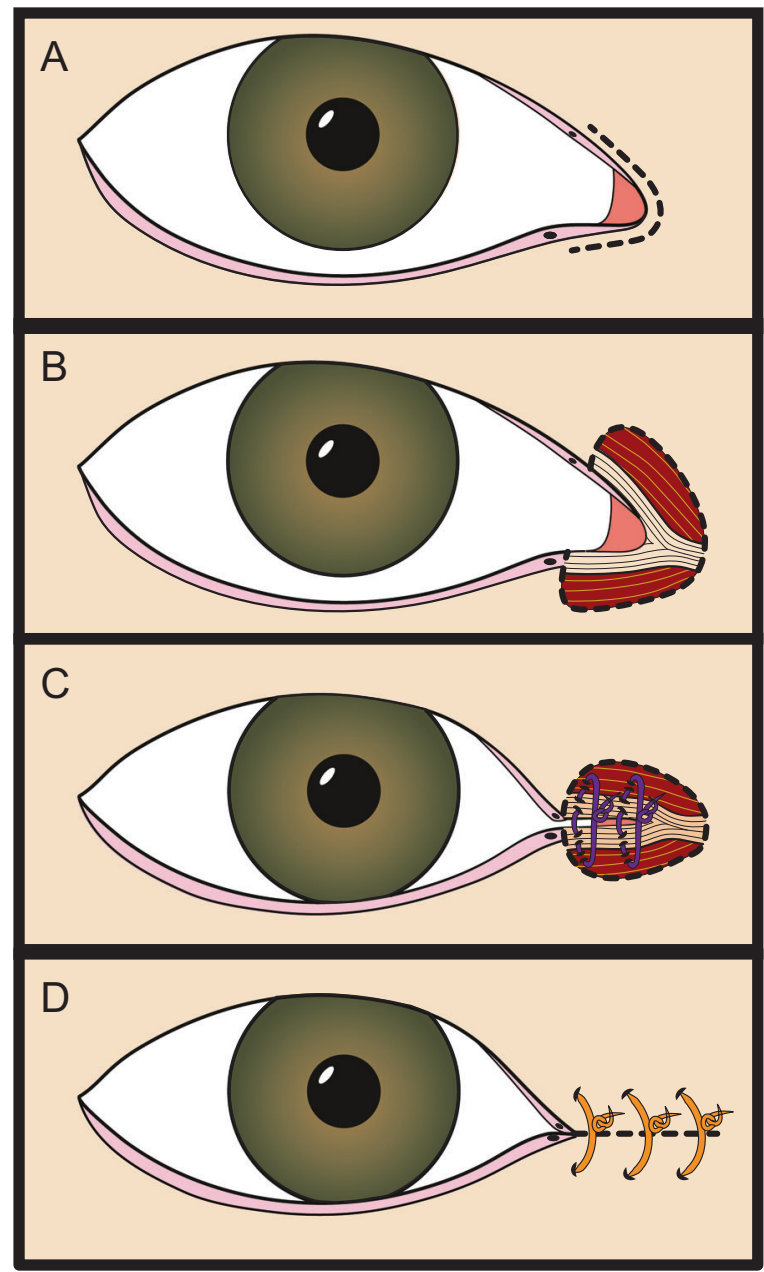

Fig. 1 Surgical technique. a An incision is created at the mucocutaneous junction medial to the upper and lower puncta. b Undermining of the skin flaps results in upper and lower medial canthal tendon exposure. c The upper and lower canthal tendons are adjoined with interrupted polyglactin sutures. d The skin flaps are closed with interrupted gut sutures

eyelid retraction, facial nerve palsy, keratoconjunctivitis sicca, and neurotrophic keratopathy (Table 1). Causes of lower eyelid retraction were further subcategorized, and included anatomical variants with prominent globes and flat cheeks $(n=44)$, eyelid laxity $(n=4)$, prior cosmetic surgery $(n=4)$, prior functional surgery $(n=3)$, and thyroid eye disease $(n=2)$.

Complete or partial improvement in ocular symptoms was observed in 95\% (69/73) of cases (Table 2). Of the 48 eyes with pre-operative lagophthalmos on examination, $85 \%$ (41/48) demonstrated reduction or resolution of lagophthalmos. Furthermore, pre-operative lower eyelid retraction, ectropion, and laxity was corrected in 95\% (69/ 73) of the cases. An improvement in corneal keratopathy at follow-up examination was observed in $91 \%(60 / 67)$ at the final follow-up visit. Visual acuity improved by at least one line in $45 \%$ of cases (33/73) and remained stable in $41 \%$ of 
Table 1 Indications for the medial canthoplasty

\begin{tabular}{ll}
\hline Lower eyelid retraction & $\mathbf{5 7}$ \\
Anatomical variant & 44 \\
Eyelid laxity & 4 \\
Prior cosmetic surgery & 4 \\
Prior functional surgery & 3 \\
Thyroid eye disease & 2 \\
Facial nerve palsy & $\mathbf{1 1}$ \\
Iatrogenic & 8 \\
Congenital & 2 \\
Other & 1 \\
Keratoconjunctivitis sicca & $\mathbf{2}$ \\
Neurotrophic keratopathy & $\mathbf{3}$ \\
Total & $\mathbf{7 3}$ \\
\hline
\end{tabular}

Table 2 Outcomes using the medial canthoplasty technique

\begin{tabular}{|c|c|c|c|c|c|c|c|c|}
\hline & \multicolumn{2}{|c|}{$\begin{array}{l}\text { Eyelid } \\
\text { Retrac- } \\
\text { tion }\end{array}$} & \multicolumn{2}{|c|}{$\begin{array}{l}\text { Facial } \\
\text { Palsy }\end{array}$} & \multicolumn{2}{|c|}{ Other } & \multicolumn{2}{|c|}{ Total } \\
\hline & $n$ & $\%$ & $n$ & $\%$ & $n$ & $\%$ & $n$ & $\%$ \\
\hline \multicolumn{9}{|l|}{ Symptoms } \\
\hline Total with any improvement & 56 & 98 & 9 & 82 & 4 & 80 & 69 & 95 \\
\hline Fully resolved & 47 & 82 & 5 & 45 & 1 & 20 & 53 & 73 \\
\hline Partial improvement & 9 & 16 & 4 & 36 & 3 & 60 & 16 & 22 \\
\hline Unchanged & 1 & 2 & 2 & 18 & 1 & 20 & 4 & 5 \\
\hline Worsening & 0 & 0 & 0 & 0 & 0 & 0 & 0 & 0 \\
\hline \multicolumn{9}{|l|}{ Ocular medication usage } \\
\hline Decreased & 19 & 33 & 3 & 27 & 1 & 20 & 23 & 32 \\
\hline Unchanged & 31 & 54 & 6 & 55 & 1 & 20 & 38 & 52 \\
\hline Increased & 7 & 12 & 2 & 18 & 3 & 60 & 12 & 16 \\
\hline \multicolumn{9}{|l|}{ Visual acuity } \\
\hline Improved & 26 & 46 & 5 & 45 & 2 & 40 & 33 & 45 \\
\hline Unchanged & 24 & 42 & 4 & 36 & 2 & 40 & 30 & 41 \\
\hline Worsened & 7 & 12 & 2 & 18 & 1 & 20 & 10 & 14 \\
\hline \multicolumn{9}{|l|}{ Lagophthalmos } \\
\hline Total with any improvement & 32 & 91 & 7 & 64 & 2 & 100 & 41 & 85 \\
\hline Fully resolved & 28 & 80 & 4 & 36 & 2 & 100 & 34 & 71 \\
\hline Partial improvement & 4 & 11 & 3 & 27 & 0 & 0 & 7 & 15 \\
\hline Unchanged & 2 & 6 & 2 & 18 & 0 & 0 & 4 & 8 \\
\hline Worsening & 1 & 3 & 2 & 18 & 0 & 0 & 3 & 6 \\
\hline \multicolumn{9}{|l|}{ Corneal findings ${ }^{\mathrm{a}}$} \\
\hline Total with Any Improvement & 49 & 94 & 9 & 90 & 2 & 40 & 60 & 90 \\
\hline Full resolution of SPEE & 30 & 58 & 5 & 50 & 2 & 40 & 37 & 55 \\
\hline Reduction of SPEE & 19 & 37 & 4 & 40 & 0 & 0 & 23 & 34 \\
\hline No change & 3 & 6 & 1 & 10 & 3 & 60 & 7 & 10 \\
\hline Worsened & 0 & 0 & 0 & 0 & 0 & 0 & 0 & 0 \\
\hline
\end{tabular}

${ }^{\text {a Patients without ocular surface disease both pre- and post-operatively }}$ were not included. SPEE Superficial Punctate Epithelial Erosions the cases (30/73). Of note, the remaining 10 patients were found to have decreased post-operative visual acuity as a result of cataract maturation (8/10) and corneal scarring due to unrelated herpetic keratitis (1/10) and bacterial ulcer formation (1/10). Finally, fewer ocular drops or ointments were required postoperatively in $32 \%$ (23/73).

Post-operatively, no surgical infections were identified. One surgical complication was reported, consisting of a surgical site wound dehiscence, which was revised without any long-term complications. Obscuration of peripheral vision as a result of the medial canthoplasty was not reported. There were no incidents of intraoperative surgical damage to the canaliculi, and new-onset, post-operative tearing and epiphora was reported following one unilateral case. Only 5.5\% (4/73) of cases went on to have a subsequent procedure (lateral tarsorrhaphy: two cases; punctal cautery: two cases) to address persistent symptoms or a worsening ocular examination.

\section{Discussion}

Numerous surgical techniques have been described to address exposure keratopathy in the presence of medial eyelid laxity, ectropion, malposition, and orbicularis weakness [6-18]. Procedures such as the medial tarsal strip, medial canthal resection, and methods for anterior and posterior medial canthal tendon tightening are primarily designed to address lower eyelid laxity related to medial canthal tendon laxity and dehiscence. These techniques may also vertically narrow the palpebral fissure by elevating the lower eyelid. The medial canthoplasty described in this report narrows the fissure both vertically and horizontally, in addition to providing medial lower eyelid support. Furthermore, this procedure may provide added benefit due to functional lacrimal obstruction and impairment of the lacrimal pump mechanism that result from canalicular apposition, potentially decreasing tear flow and increasing tear volume. However, the medial canthoplasty does not address significant horizontal laxity and should be combined with a procedure such as the lateral tarsal strip if indicated.

Mauriello and Mostafavi [19] previously described the successful use of the medial canthoplasty in combination with other procedures in 14 patients with lagophthalmos and lower eyelid laxity. In the present study, we evaluated cases that utilized the medial canthoplasty as a standalone intervention to limit the confounding effects of the simultaneous surgeries such as eyelid tightening procedures and upper eyelid weight placement. The majority of isolated medial canthoplasty cases were performed to address lower eyelid retraction (78\%; 57/73), and $98 \%$ of this group reported post-operative symptom improvement, 91\% 


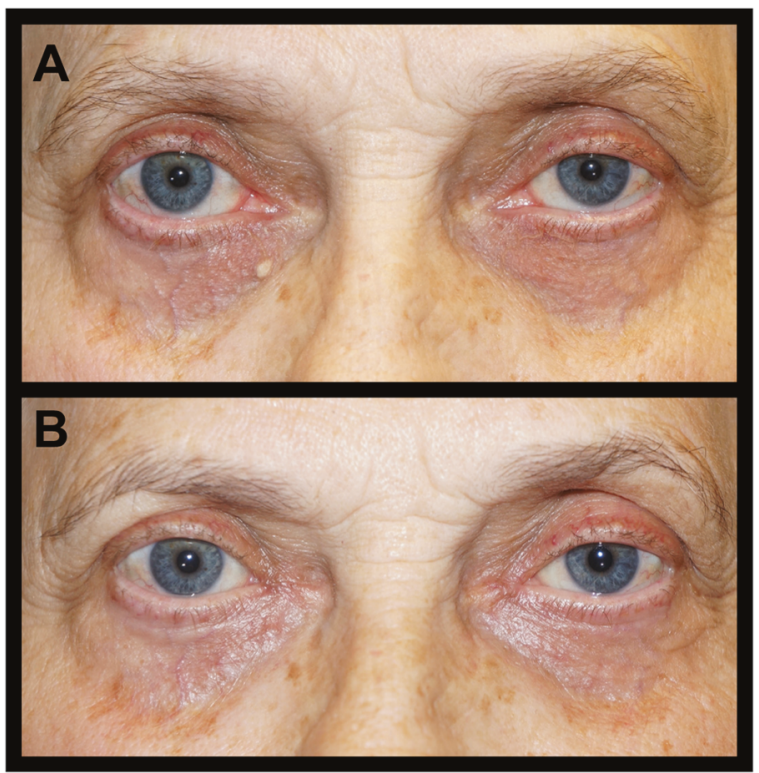

Fig. 2 Representative photographs of a patient with bilateral lower eyelid retraction. a Pre-operative photograph. Note the inferior scleral show and increased medial canthal exposure present bilaterally. b Post-operative photograph. Following bilateral treatment with medial canthoplasty, the patient demonstrates narrowing of the vertical fissure with reduced inferior scleral show and a subtle decrease in horizontal palpebral length, providing improved medial eyelid support and diminished ocular exposure nasally

demonstrated a reduction in lagophthalmos, and 94\% showed improved corneal surface findings. Based on the clinical and patient-reported improvement observed in these cases, we believe the medial canthoplasty may be utilized as a satisfactory single intervention in the presence of mild to moderate lower eyelid retraction (Fig. 2). Interestingly, the few cases in which the medial canthoplasty was used as a standalone procedure to treat facial palsy also showed exceptional improvement in symptoms $(82 \% ; 9 / 11)$ and corneal keratopathy $(90 \% ; 9 / 10)$. However, it is important to note that this subgroup treated with only a medial canthoplasty demonstrated only mild pre-operative lagophthalmos (10/11 with lagophthalmos $\leq 5 \mathrm{~mm}$ ), and patients with more severe lagophthalmos were generally treated with additional simultaneous procedures and excluded from this study. Although the limited sample size of this facial palsy subgroup prevents any definitive conclusions, the finding suggests that the medial canthoplasty may be a suitable standalone treatment option in patients with mild lagophthalmos, where as more severe cases could benefit from combined techniques.

Limitations of this study include the retrospective design, limited availability of eyelid measurements (i.e. marginal reflex distance-2; measured scleral show), and a lack of control group. One limitation of the medial canthoplasty that is important to discuss with patients prior to surgery is the modest change medial canthal angle appearance that results from horizontal palpebral shortening. However, since the upper and lower puncta are located only 5-6 mm from the canthal angle, fusion of this short segment of the medial eyelid results in minimal cosmetic change. Furthermore, when compared to medial and lateral tarsorrhaphies, the medial canthoplasty may provide a more esthetically favorable outcome with no reported reduction in visual field.

In summary, the medial canthoplasty is a safe, simple, and effective procedure that narrows the palpebral fissure and provides support for the lower eyelid with an acceptable esthetic outcome. The medial canthoplasty procedure is uncomplicated and requires minimal operative time, allowing it to be used as a standalone procedure to treat exposure keratopathy caused by lower eyelid retraction and facial palsy.

\section{Summary}

\section{What was known before}

- Exposure keratopathy caused by lower eyelid retraction, facial nerve palsy, and previous periocular surgery can result in significant ocular complications. Many surgical techniques have been described to address the causes of exposure keratopathy, including the medial canthoplasty. While other variations of the medial canthoplasty have previously been reported, the literature describing long-term outcomes remains limited.

\section{What this study adds}

- This study aims to report the functional outcomes of the medial canthoplasty as a standalone surgical intervention for management of exposure keratopathy. In this study, approximately $90 \%$ of these cases resulted in improved clinical findings as well as patient-reported ocular symptoms.

\section{Compliance with ethical standards}

Conflict of interest The authors declare that they have no conflict of interest.

Publisher's note: Springer Nature remains neutral with regard to jurisdictional claims in published maps and institutional affiliations.

\section{References}

1. Lane C. Management of ocular surface exposure. Br J Ophthalmol. 2012;96:471-2. 
2. Chi JJ. Management of the eye in facial paralysis. Facial Plast Surg Clin North Am. 2016;24:21-8.

3. Piskiniene R. Eyelid malposition: lower lid entropion and ectropion. Medicine (Kaunas). 2006;42:881-4.

4. Hahn S, Desai SC. Lower lid malposition: causes and correction. Facial Plast Surg Clin North Am. 2016;24:163-71.

5. Pereira MV, Glória AL. Lagophthalmos. Semin Ophthalmol. 2010;25:72-8.

6. Bodian M. A simple operation for lateral tarsorrhaphy. Arch Ophthalmol. 1965;74:74-6.

7. Anderson RL, Gordy DD. The tarsal strip procedure. Arch Ophthalmol. 1979;97:2192-6.

8. Nowinski TS, Anderson RL. The medial spindle procedure for involutional medial ectropion. Arch Ophthalmol. 1985;103:1750-3.

9. Ritleng P. Medial canthoplasty for treatment of severe lacrimal ectropion. Orbit. 1989;8:49-56.

10. Jordan DR, Anderson RL, Thiese SM. The medial tarsal strip. Arch Ophthalmol. 1990;108:120-4.

11. Sullivan TJ, Collin JR. Medical canthal resection: an effective long-term cure for medial ectropion. $\mathrm{Br} \quad \mathrm{J}$ Ophthalmol. 1991;75:288-91.

12. Tanenbaum M, Gossman MD, Bergin DJ, et al. The tarsal pillar technique for narrowing and maintenance of the interpalpebral fissure. Ophthalmic Surg. 1992;23:418-25.
13. Castroviejo-Bolibar M, de Damborenea A, Fernández-Vega A. Surgical repair of paralytic lagophthalmos by medial tarsal suspension of the lower lid. Br J Ophthalmol. 1996;80:708-12.

14. Fante RG, Elner VM. Transcaruncular approach to medial canthal tendon plication for lower eyelid laxity. Ophthal Plast Reconstr Surg. 2001;17:16-27.

15. Frueh BR, Su CS. Medial tarsal suspension: a method of elevating the medial lower eyelid. Ophthal Plast Reconstr Surg. 2002;18:133-7.

16. Demirci H, Hassan AS, Elner SG, et al. Comprehensive, combined anterior and transcaruncular orbital approach to medial canthal ligament plication. Ophthal Plast Reconstr Surg. 2007;23:384-8.

17. Tao JP, Vemuri S, Patel AD, Compton C, Nunery WR. Lateral tarsoconjunctival onlay flap lower eyelid suspension in facial nerve paresis. Ophthal Plast Reconstr Surg. 2014;30:342-5.

18. Lee OS. An operation for the correction of everted lacrimal puncta. Am J Ophthalmol. 1951;34:575-8.

19. Mauriello JA Jr, Mostafavi R. Medial canthoplasty for optimum support of the lower eyelid in 14 patients. Ophthalmic Surg Lasers. 1996;27:869-75.

20. Lisman RD, Smith B, Baker D, Arthurs B. Efficacy of surgical treatment for paralytic ectropion. Ophthalmology. 1987;94:671-81. 